Regards sur l'économie allemande

Bulletin économique du CIRAC

$65 \mid 2004$

Varia

\title{
Emploi : difficile mise en œuvre des réformes Hartz
}

\section{Isabelle Bourgeois}

\section{OpenEdition}

Journals

Édition électronique

URL : http://journals.openedition.org/rea/3951

DOI : 10.4000/rea.3951

ISBN : 978-2-8218-0826-3

ISSN : 1965-0787

Éditeur

CIRAC

Édition imprimée

Date de publication : 1 mars 2004

Pagination : 36-38

ISSN : 1156-8992

Référence électronique

Isabelle Bourgeois, «Emploi : diffıcile mise en œuvre des réformes Hartz », Regards sur l'économie allemande [En ligne], 65 | mars 2004, mis en ligne le 29 octobre 2009, consulté le 15 septembre 2020. URL : http://journals.openedition.org/rea/3951

Ce document a été généré automatiquement le 15 septembre 2020

(C) CIRAC 


\title{
Emploi : difficile mise en œuvre des réformes Hartz
}

\author{
Isabelle Bourgeois
}

\section{La faillite de Maatwerk révèle...}

1 La déclaration de faillite, déposée le 16 février par l'agence d'intérim Maatwerk (Hambourg), ravive la polémique sur la mesure-phare des réformes « Hartz I » adoptées en 2002: la mise en place des Personal-Service-Agenturen (PSA). Ces «agences de service de personnel ", créées depuis avril 2003, ont pour mission de réinsérer durablement (en CDI) des chômeurs difficiles à placer que leur envoient les ANPE allemandes. En contrepartie, ces dernières versent aux PSA un forfait mensuel moyen de $1200 €$ par personne, destiné à couvrir partiellement leurs charges. Car pour pouvoir placer les chômeurs qui leur sont confiés, les PSA doivent d'abord les embaucher et les requalifier. L'objectif de cette mesure entrée en vigueur au $1^{\mathrm{er}}$ janvier 2003 était de réintégrer en un an quelque 50000 chômeurs. Or depuis leur mise en place, les PSA n'ont accueilli que 15300 personnes, dont elles n'ont pu placer en CDI que 6375.

\section{... que les PSA sont peu rentables}

2 L'insolvabilité de Maatwerk repose aujourd'hui avec d'autant plus d'acuité la question de l'efficience de cette forme d'intérim subventionné par l'Etat que le coût de cette seule mesure ( 175 millions $€$ ) semble quelque peu disproportionné aux attentes et, surtout, à la réalité. Et, face aux impératifs de consolidation budgétaire, certains se demandent s'il ne vaudrait pas mieux revenir à des mesures plus classiques de traitement social du chômage, nettement moins onéreuses. La Fédération allemande de l'intérim Bundesverband Zeitarbeit (BZA) estime par ailleurs que d'autres faillites devraient suivre, car les activités d'une PSA sont difficilement rentables. En tant qu'employeur des chômeurs à placer, elles doivent les rémunérer même entre deux 
missions; or cette rémunération est à peu de choses près identique au salaire conventionnel en vigueur dans chaque branche, ce qui réduit nettement les marges des PSA prestataires quand elles parviennent à conclure avec une entreprise cliente, et n'incite pas non plus les entreprises à recourir aux services d'une PSA. Autrement dit : la mesure a surtout permis à la fois d'éluder la question de l'ouverture d'un segment à bas salaires pour les moins qualifiés et de déréguler à outrance le marché de l'intérim (voir REA 62/03) en arguant du fait que l'intérim (subventionné) est un outil d'insertion.

\section{Maatwerk : une erreur d'appréciation du marché}

3 Mais les distorsions de concurrence que redoutait la Fédération BZA ne se sont pas manifestées, les lois du marché ayant au contraire amorcé la restructuration des PSA. Dans le cas de Maatwerk, filiale allemande de la société néerlandaise du même nom, les observateurs relèvent deux erreurs notables. La première est le fait de l'entreprise et peut se résumer à une erreur stratégique d'appréciation du marché allemand, sur laquelle ses concurrents avaient pourtant attiré son attention. Maatwerk avait implanté son réseau outre-Rhin sans disposer de ce dense réseau de contacts dans le tissu régional d'entreprises sans lequel le placement de chômeurs réputés 'difficiles' est pratiquement impossible. Et, de fait, Maatwerk avait un taux de placement $(10 \%)$ nettement inférieur à la moyenne des PSA (40\%).

\section{Nuremberg : trop de licences attribuées à une seule société}

La seconde erreur est le fait de l'Agence fédérale pour l'emploi (nouveau nom de l'office fédéral du Travail) qui avait attribué à Maatwerk 201 licences, soit un cinquième du total de 985. Car les PSA sont soumises au régime des licences; et Maatwerk, spécialiste hollandais de l'insertion de Rmistes, facturait ses prestations à bas prix. Ses principaux concurrents, les grands du travail temporaire 'classique', s'étaient montrés plus prudents en se diversifiant dans ce créneau: BBJ Consult (46 PSA), Randstad (22) ou Manpower (12).

\section{Une « affaire » de plus pour l'Office?}

5 Cela n'est pas de nature à redorer l'image de l'office de Nuremberg, secoué par diverses «affaires » depuis que, début 2002, un rapport de la Cour des comptes fédérale avait mis en doute sa gestion (voir REA 55/02). La Cour avait révélé de graves irrégularités dans la gestion des statistiques relatives au placement des chômeurs et incité pouvoirs publics et partenaires sociaux à moderniser cette institution. Le président de l'office, Bernd Jagoda, avait été contraint à la démission. Quant à son successeur, Florian Gerster, le ministre fédéral de l'Economie avait mis prématurément fin à son contrat le 24 janvier 2004. Cet ancien ministre des Affaires sociales de Rhénanie-Palatinat, nommé pour mener à bien la restructuration de l'office, avait défrayé la chronique pour avoir passé divers marchés d'audit et de conseil sans procédure d'appel d'offres, notamment avec le cabinet Roland Berger dont le président figurait parmi les membres de la Commission Hartz. 


\section{Un « mammouth » en restructuration}

Son successeur, Franck-Jürgen Weise, désigné le 6 février 2004 par le conseil d'administration de l'office, devra poursuivre la difficile modernisation d'un «mammouth» (son surnom en Allemagne) aux effectifs pléthoriques: 99779 très exactement, dont 22100 fonctionnaires et 67963 employés de la fonction publique. Dès son arrivée, Florian Gerster avait profondément choqué en déclarant vouloir le " dégraisser ». Cet objectif figure pourtant parmi ceux des lois « Hartz II + III » qui visent tous à accroître l'efficience d'une administration de l'échelon fédéral qui, statutairement, se substitue au Bund pour gérer, en pleine autonomie, l'ensemble de la politique en faveur de l'emploi.

\section{Redéfinition des missions}

7 Cette collectivité de droit public de l'échelon fédéral ne change pas de statut, mais s'est vu confier de nouvelles missions : celles d'un prestataire de services de placement des ressources humaines. Pour le concrétiser, l'office avait été rebaptisé Agence (Bundesagentur für Arbeit). Sa modernisation, engagée depuis 2003, suit plusieurs axes. En interne: un meilleur suivi des dossiers. Ainsi, un agent 'gère' aujourd'hui 350 chômeurs au lieu de 800 auparavant. Cela s'est traduit par une hausse du nombre des agents chargés du placement, passé de 4000 à 12 000. En contrepartie, les effectifs de la 'holding' de Nuremberg ont été réduits de 1100 à 400. En externe, l'accent est mis sur la culture client. Pour l'instant, dans 10 sites pilotes seulement, chargés de mieux faire se rencontrer sur le terrain l'offre et la demande. Cela implique une meilleure écoute des entreprises et un suivi individualisé des chômeurs. Mais on y teste surtout la séparation des deux principales fonctions réunies au sein de l'Agence : le placement des chômeurs et la gestion des revenus de substitution.

\section{Réduction des dépenses liées aux « mesures »}

Au plan budgétaire, enfin, elle est appelée à réduire ses dépenses relatives aux mesures actives en faveur de l'emploi. Avec 56,99 milliards $€$, son budget prévisionnel 2004 est sensiblement identique aux deux exercices précédents. Mais sa ventilation diffère quelque peu: les dépenses programmées au titre de l'Arbeitslosengeld s'élèvent à 29,5 milliards $€$, une augmentation qui reflète la hausse du chômage. A l'inverse, le chapitre des mesures actives baisse de 1,37 milliards pour s'établir à 20,16 milliard $€$. Côté recettes, le total est inférieur de 5,21 milliards $€$ aux dépenses - un déficit que comble le gouvernement fédéral. Les recettes fiscales, versées par les divers échelons de collectivités territoriales pour financer une large partie des "mesures", sont en effet en baisse. Quant au volume des cotisations sociales, affecté par les tensions sur le marché de l'emploi, il passe de 49,4 milliards $€$ à 48,3 milliards $€$. La baisse est significative en comparaison de la hausse du volume de l'Arbeitslosengeld financé par l'assurance chômage. Reste à savoir dans ce contexte comment l'Agence compte affronter la gestion de l'Arbeitslosengeld II, né de la fusion entre aide au chômeur et aide sociale, qui lui échoit dans le cadre des réformes adoptées à la fin 2003. 
Dépenses de l'Agence fédérale pour l'Emploi (en millions $€$ )

\begin{tabular}{|l|l|l|}
\hline & 2002 & 2003 \\
\hline Allocations chômage (Arbeitslosengeld) & 27007 & 29048 \\
\hline Frais généraux de gestion & 4225 & 4421 \\
\hline Autres mesures en faveur de l'emploi & 3306 & 3689 \\
\hline Soutien aux handicapés & 3101 & 3296 \\
\hline Soutien à la qualification et à la formation professionnelle des jeunes & 3214 & 3131 \\
\hline Allocations de formation continue ${ }^{*}$ ) & 3997 & 2972 \\
\hline Mesures de création d'emplois & 3168 & 2333 \\
\hline Formation continue (hors Unterhaltsgeld) & 2705 & 2029 \\
\hline Autres revenus de substitution & 2552 & 1913 \\
\hline Soutien aux créateurs d'entreprise & 1006 & 1681 \\
\hline Subventions salariales & 1645 & 1590 \\
\hline Frais divers & 582 & 572 \\
\hline Personal-Service-Agenturen & 56508 & 56850 \\
\hline Total & 175 \\
\hline & - & 329 \\
\hline
\end{tabular}

Source des données : Bundesagentur für Arbeit (www.arbeitsagentur.de), iwd n 6/2004. *) Unterhaltsgeld versé aux chômeurs en requalification (67 \% du salaire net).

\section{Sur le long terme : une modernisation « culturelle »}

9 La modernisation du «mammouth » prendra beaucoup de temps, expliquait Franck-J. Weise alors qu'il était encore son directeur financier; elle se heurte avant tout à un problème culturel. Car «ses structures sclérosées, la rigidité des hiérarchies et des voies administratives inhibent toute créativité chez ses collaborateurs ». Mais le fond $\mathrm{du}$ problème est structurel : le fonctionnement tripartite de l'Agence est vivement critiqué pour les dérives corporatistes auxquelles il a mené. Et pour ce paradoxe : ceuxlà mêmes qui, en régulant le marché de l'emploi, privilégient les insiders (ceux qui sont en emploi), au détriment d'un nombre croissant d'outsiders (les chômeurs), régulent également la politique de lutte contre le chômage via la détermination et la gestion des revenus de substitution. C'est là l'une des origines du haut niveau de chômage structurel outre-Rhin. Mais aucune des parties impliquées n'a jusqu'ici abordé 
franchement cette question dans le cadre des vastes réflexions sur la modernisation de l'emploi. (IB)

INDEX

Mots-clés : emploi, travail, marché du travail, réformes Hartz, réforme, budget, finances publiques, politique sociale, politique de l'emploi, revenu 